\title{
Valve-sparing versus composite root replacement procedures in patients with Marfan syndrome
}

\author{
Joseph S. Coselli ${ }^{1,2,3}$, Scott A. Weldon ${ }^{1,4}$, Ourania Preventza ${ }^{1,2,3}$, Kim I. de la Cruz ${ }^{1,2,3}$, Scott A. LeMaire , $^{1,2,3}$ \\ ${ }^{1}$ Division of Cardiothoracic Surgery, Michael E. DeBakey Department of Surgery, Baylor College of Medicine, Houston, Texas, USA; ${ }^{2}$ Section of Adult \\ Cardiac Surgery, Department of Cardiovascular Surgery, Texas Heart Institute, Houston, Texas, USA; ${ }^{3}$ CHI St. Luke's Health-Baylor St. Luke's Medical \\ Center, Houston, Texas, USA; ${ }^{4}$ Surgical Research Core, Michael E. DeBakey Department of Surgery, Baylor College of Medicine, Houston, Texas, USA \\ Correspondence to: Scott A. LeMaire, MD. One Baylor Plaza, BCM 390, Houston, TX 77030, USA. Email: slemaire@bcm.edu.
}

Submitted Jul 28, 2017. Accepted for publication Oct 26, 2017.

doi: $10.21037 /$ acs.2017.11.02

View this article at: http://dx.doi.org/10.21037/acs.2017.11.02

\section{Introduction}

Marfan syndrome (MFS) is a heritable thoracic aortic disease that has devastating effects on the ocular, skeletal, and cardiovascular systems. A grossly dilated aortic root is the signature manifestation of cardiovascular pathology in MFS (Figure 1); the root is also the primary location of aortic dissection and rupture that have historically ended the lives of these patients prematurely. In 1968, Bentall and De Bono (1) devised an approach to performing comprehensive aortic root replacement (ARR) in a 33-yearold man with presumed MFS by using a mechanical valve in combination with a graft. During the past two decades, two competing approaches have been established for performing ARR in patients with MFS: valve-sparing and valve-replacing approaches. In recent years, valve-sparing approaches have become increasingly attractive to both patients and clinicians because, if patients keep their own aortic valve leaflets, they can avoid the life-long regimen of anticoagulation that is necessitated by mechanical valve replacement. Often, whether to perform a valve-sparing or a valve-replacing aortic root repair is determined intraoperatively upon inspection of the aortic valve leaflets. Aortic valve replacement may be preferable in some cases, such as when leaflets have large fenestrations or heavy calcification and scarring.

Among the centers experienced in ARR, the methods used to perform these repairs can differ substantially, particularly with respect to the diameter of the replacement graft, the use of a straight "tube" graft versus one with neosinuses, and the number of subannular sutures $(3,6,12$, or more) necessitated during root replacement (2). Here, we describe our techniques used for performing ARR in patients with MFS. In the first case, we used a valve-sparing approach in a 41-year-old woman; in the second case, we used a valve-replacing approach in a 28 -year-old man.

\section{Case 1: valve-sparing ARR}

\section{Clinical vignette}

A 41-year-old woman with MFS underwent extensive distal aortic repair (namely, a Crawford extent II thoracoabdominal aortic aneurysm repair) necessitated by the dilatation of a chronic DeBakey type III aortic dissection, which occurred 5 years previously. Four months after the distal aortic repair, the patient returned for elective proximal aortic repair. Her aortic root was mildly dilated to a diameter of $4.5 \mathrm{~cm}$, which tapered to a normal aortic diameter of $2.2 \mathrm{~cm}$ in the distal aspect of the ascending aorta and proximal portion of the transverse aortic arch (Figure $2 A$ ). The annuloaortic ectasia pattern of dilatation was consistent with MFS. Trace aortic valve regurgitation was present. Noteworthy comorbidities included a greatly reduced left ventricular ejection fraction of $<20 \%$ and severe global left ventricular hypokinesis. ARR was indicated; because of the patient's age and preference, a valve-sparing approach to ARR was desired.

\section{Operative technique}

The patient's chest was opened by using a standard median sternotomy. After a partial occluding clamp was placed on the innominate artery, an $8-\mathrm{mm}$ graft was anastomosed 
to the vessel as a site for cardiopulmonary bypass inflow. The patient was cooled to $30{ }^{\circ} \mathrm{C}$, and the ascending aorta was cross-clamped proximal to the innominate artery. The aneurysm was opened at the sinotubular junction, the

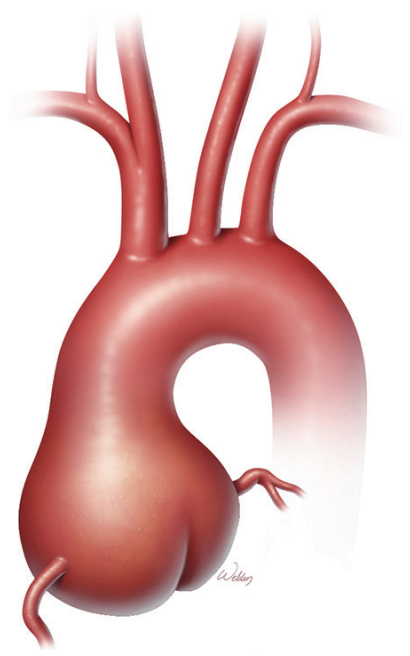

Figure 1 Illustration of annuloaortic ectasia, the distinctive aortic root dilatation that is commonly present in patients with Marfan syndrome. (Used with permission of Baylor College of Medicine.) diseased aortic root tissue (including the sinuses of Valsalva) was excised, and the coronary arteries were mobilized on buttons of aortic tissue. Antegrade and retrograde cardioplegia were provided intermittently throughout the procedure.

The aortic valve leaflets were examined and were found to be noticeably thin (consistent with MFS) and contained some small minor fenestrations; however, the leaflets were reasonably competent and were deemed suitable for a valve-sparing approach to ARR. A 28-mm Valsalva graft (Vascutek $^{\circledR}$ Gelweave Valsalva ${ }^{\mathrm{TM}}$ graft, Vascutek Ltd., a subsidiary of Terumo Corporation, Renfrewshire, Scotland) was selected by measuring the annulus with a valve sizing ring. As an alternative, the graft size can be selected by measuring the distance between the lower part of the valve annulus and the peak of the commissure between the left and noncoronary cusps. One should be mindful that the residual tissue of the valve apparatus inside the graft tends to narrow the aortic root complex by 2 to $3 \mathrm{~mm}$ (for example, a $28-\mathrm{mm}$ graft may ultimately have a $24-$ to $25-\mathrm{mm}$ orifice).

Six 2-0 polypropylene mattress sutures with felt pledgets were placed in the subannular position to anchor the graft;
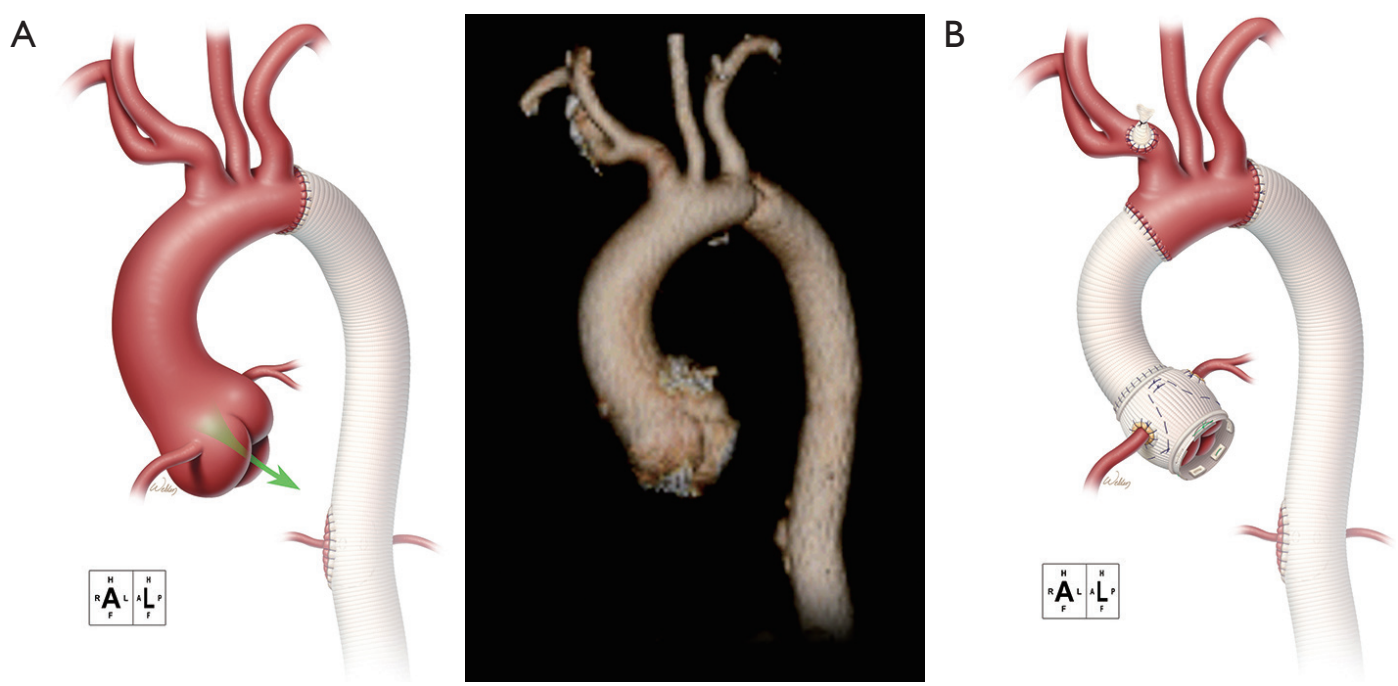

Figure 2 Preoperative anatomy and postoperative aortic reconstruction for Case 1. Preoperative anatomy (A). Illustration and reconstruction of a computed tomography scan showing the patient's mildly dilated aortic root (which tapered to a normal aortic diameter in the distal ascending aorta and transverse aortic arch), as well as a portion of the patient's prior extent II thoracoabdominal aortic aneurysm repair. Postoperative aortic reconstruction (B). An illustration of the completed aortic root reconstruction and replacement of the ascending aorta is shown. A valve-sparing approach to aortic root replacement was used, and the coronary arteries were reattached as buttons. The suture line between the Valsalva graft (containing the reconstructed aortic root) and the tube graft is shown. (Used with permission of Baylor College of Medicine.) 
the sutures were brought from the inside of the valve to the outside. Pledgets help secure the graft and may prevent a fistula from developing in the region of the junction between the noncoronary sinus and the right coronary sinus. The graft was soaked in rifampin solution $(600 \mathrm{mg} / \mathrm{vial})$, and a small notch was made in the graft to accommodate the difference in height on the commissure between the right and left coronary cusps. The subannular sutures were then placed through the proximal portion of the Valsalva graft, and the distal graft was trimmed just above the neo-sinus portion for improved access when reconstructing the valve. The graft was then parachuted into place, and the sutures were tied. Although the sutures were not placed under great tension, they slightly reduced the graft's diameter.

The valve apparatus was brought up into the graft by using 4-0 polypropylene suture, and the peaks of the commissures were brought up high on the graft so that the valve apparatus was elevated within the graft. Notably, the coaptation of valve leaflets should be at a plane that is higher than the valve annulus. Beginning at the nadir of each commissure, a 4-0 polypropylene suture was run up to the top of the commissure in a baseball-stitch (i.e., inand-out) fashion. We placed felt pledgets at the nadir of each sinus, a technique that provides additional support in patients with MFS. Also, in patients with MFS, suture bites should be taken at the aortic valve annulus, which has stronger tissue. Water was placed into the reconstructed root to test the coaptation of the aortic valve leaflets.

After a "lifesaver" of autogenous pericardium was placed around the origin of the mobilized left coronary artery for reinforcement, the artery was then reattached by using a running 6-0 polypropylene suture to a small hole in the neo-sinus of the graft that was made with cautery; the amount of residual aortic tissue around the artery was minimized without compromising its origin.

Because the distal aspect of the ascending aorta and the transverse aortic arch were of normal diameter, a rifampinsoaked, $20-\mathrm{mm}$ tube graft was selected to replace the ascending aorta. Using a running 4-0 polypropylene suture, we anastomosed the tube graft to the end of the distal ascending aorta. Additionally, we reinforced the anastomosis with felt pledgets by using 4-0 polypropylene mattress sutures. The tube graft was then cut to appropriate length and anastomosed end-to-end to the Valsalva graft by using a running 4-0 polypropylene suture, forming a reconstructed sinotubular junction. Using a running 6-0 polypropylene suture, the right coronary artery was placed high upon the neo-sinus over a small hole made with cautery, and, similar to the reconstruction of the left coronary artery, reattachment was reinforced with autogenous pericardium. The completed repair is shown in Figure $2 B$.

\section{Outcome}

A postoperative echocardiogram showed normal aortic valve function and improved left ventricular ejection fraction (50-55\%). The recovery period was unremarkable, and the patient was discharged home on postoperative day 6 . The patient remains well 14 months after repair.

\section{Case 2: valve-replacing ARR}

\section{Clinical vignette}

A 28-year-old man with suspected MFS was admitted to his local hospital for assessment after experiencing abdominal pain and heart palpitations. Although the patient had previously experienced a detached left retina and a pulmonary embolism, he had not undergone prior aortic or cardiac evaluation or repair. An echocardiogram revealed mild aortic valve regurgitation with aortic root dilatation and suggested a bicuspid aortic valve. A computed tomography scan confirmed a dilated aortic root $(4.6 \mathrm{~cm})$ and ascending aorta $(5.0 \mathrm{~cm})$, as well as enlargement of the proximal portion of the aortic arch $(3.3 \mathrm{~cm})$ (Figure $3 A$ ). The patient was referred to our center for further assessment. Aortic root and hemiarch replacement were indicated; because of the patient's age and preference, a valve-sparing approach to ARR was desired, if feasible.

\section{Operative technique}

The patient's chest was opened by using a standard median sternotomy. After a partial occluding clamp was placed on the innominate artery, an $8-\mathrm{mm}$ graft was anastomosed to the artery for inflow during cardiopulmonary bypass. The ascending aorta was cross-clamped proximal to the innominate artery. Antegrade and retrograde cardioplegia were provided intermittently. After the aneurysm was opened, the aortic valve leaflets were examined. Large fenestrations were seen on all 3 leaflets, and there was fusion of the right and noncoronary leaflets (Figure 3B). The aortic valve was resected after determining it was unsuitable for valve-sparing ARR. The coronary arteries were mobilized on buttons of tissue. Starting with the three commissures, 2-0 braided polyester mattress sutures with felt pledgets 

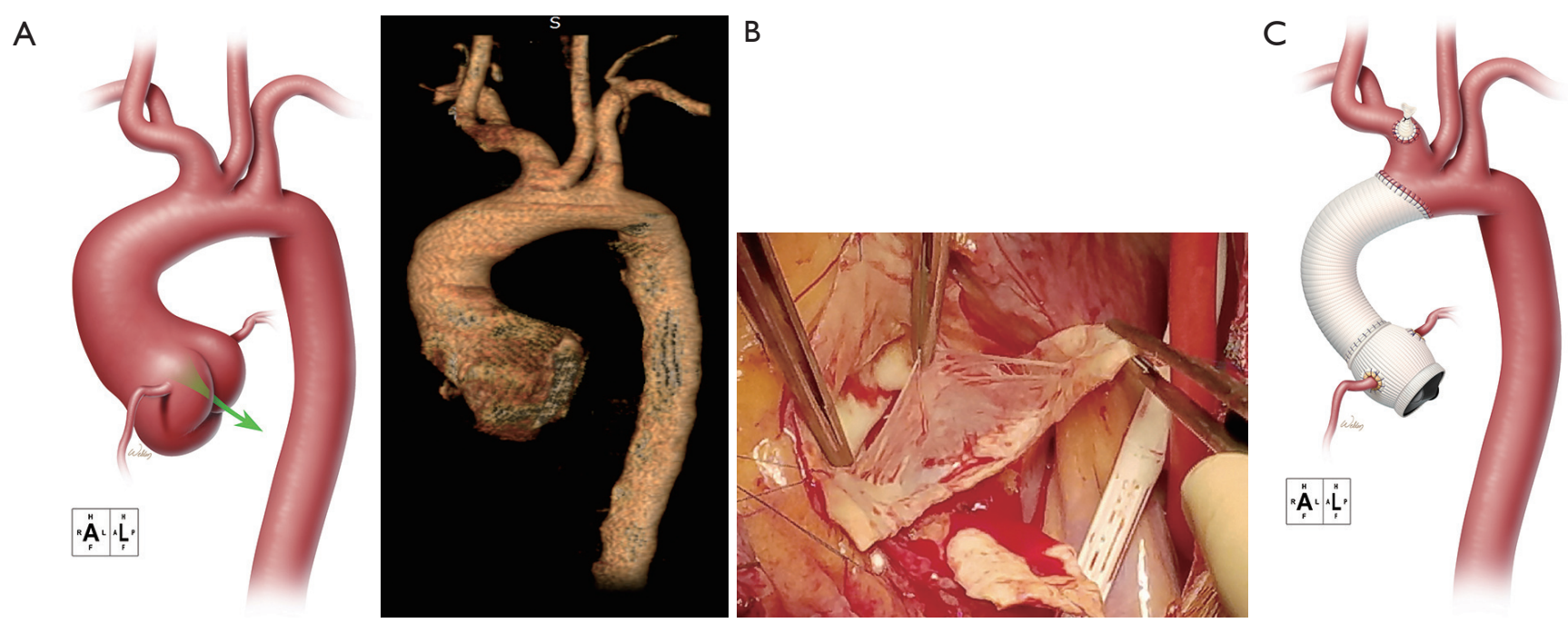

Figure 3 Preoperative anatomy and postoperative aortic reconstruction for Case 2. Preoperative anatomy (A). Illustration and reconstruction of a computed tomography scan showing the patient's dilated aortic root, ascending aorta, and proximal portion of the transverse aortic arch. Although a valve-sparing approach to aortic root replacement was desired by the patient, the native aortic valve leaflets were unsalvageable because of large fenestrations, especially at the commissures (B). Postoperative aortic reconstruction (C). An illustration of the completed aortic root reconstruction and hemiarch replacement is shown. A valve-replacing approach to aortic root replacement was used, and the coronary arteries were reattached as buttons. The suture line between the composite mechanical valve Valsalva graft and the tube graft is shown. (Used with permission of Baylor College of Medicine.)

were placed in the native annulus to anchor the composite valve graft (i.e., to perform a modified Bentall procedure). Additional annular mattress sutures with pledgets were then placed between the sutures at the commissures for a total of 15 sutures. The annulus was then sized to select an appropriate replacement valve. Once the patient was cooled to $24{ }^{\circ} \mathrm{C}$ and hypothermic circulatory arrest was initiated, the innominate artery was constricted with a Rumel tourniquet, and the pump flow was turned down to $1.5 \mathrm{~L} / \mathrm{min}$. The aortic clamp was removed, and a balloon perfusion catheter was placed into the left common carotid artery to provide bilateral antegrade cerebral perfusion. The lesser curvature of the transverse aortic arch was resected, and a rifampin-soaked, 24-mm tube graft was anastomosed end-to-end by using a running 4-0 polypropylene suture in a beveled hemiarch fashion. This anastomosis was then reinforced with 4-0 polypropylene mattress sutures with felt pledgets to ensure hemostasis. The innominate artery tourniquet was released, the balloon perfusion catheter was removed from the left common carotid artery, and flows were increased to a normal level as the graft was flushed of air and then clamped.

To replace the aortic valve and root, we used a $27-\mathrm{mm}$ composite mechanical valve Valsalva graft (Masters HPтм
Valved Graft with Gelweave Valsalva ${ }^{\mathrm{TM}}$, St. Jude Medical, St. Paul, Minnesota, USA) that was soaked in rifampin solution. The annular sutures were placed through the sewing ring of the graft, which was then parachuted into place, and the sutures were tied. The remnant aortic root tissue was sewn to the adjacent valve sewing ring with a running 3-0 polypropylene suture to provide additional hemostasis and to reduce the likelihood of a paravalvular leak.

A "lifesaver" of autogenous pericardium was created and placed around the origin of the left coronary artery, which was then reattached with a running 6-0 polypropylene suture. The composite valve graft was trimmed just distal to the neo-sinus portion, and the two grafts were anastomosed end-to-end by using a running 3-0 polypropylene suture. Using a running 6-0 polypropylene suture, we reattached the right coronary artery reinforced with autogenous pericardium. The completed repair is shown in Figure 3C.

\section{Outcome}

A postoperative echocardiogram showed normal mechanical aortic valve function. The recovery period was unremarkable. The patient began anticoagulation and was discharged home 
on postoperative day 6. The patient remains well 10 months after repair.

\section{Comments}

Near the end of the 20th century, Vince Gott and others (3) published a landmark multicenter report clearly showing that valve-replacing ARR could prolong life in MFS patients. Of interest, Gott et al. (3) reported that more than half of all valve-replacing ARRs performed were in patients with an aortic root diameter greater than $6 \mathrm{~cm}$. Importantly, by establishing that valve-replacing ARR could be safely replicated in various aortic centers and consistently result in low early mortality rates, a shift to repair at lower aortic diameters was ultimately made possible. However, most valve-replacing ARRs in patients with MFS involve replacing the valve with a mechanical prosthesis, which requires the patient to follow a lifelong regimen of anticoagulation. This limitation and the fact that most MFS patients undergoing this type of repair are typically in their mid-to-late $30 \mathrm{~s}$ created interest in developing a valve-sparing approach $(4,5)$. As evident in our multicenter publications $(6,7)$ on ARR in patients with MFS (the Aortic Valve Operative Outcomes in Marfan Patients study), valvesparing approaches are now used more commonly than are valve-replacing approaches in patients with MFS. However, as we show in the second of these two cases, there are times at which it is simply not prudent to perform valve-sparing ARR. Ultimately, the quality of the native valve leaflets determines whether valve-sparing ARR is feasible.

\section{Acknowledgements}

The authors thank Nicole Stancel, PhD, ELS; Susan Y. Green, MPH; and Hiruni S. Amarasekara, MS, for editorial support and Joseph C. Brewton for invaluable assistance with video production.

\section{Footnote}

Conflicts of Interest: Dr. Coselli serves as a consultant for Vascutek Ltd., a subsidiary of Terumo Corporation. The other authors have no conflicts of interest to declare.

\section{References}

1. Bentall H, De Bono A. A technique for complete replacement of the ascending aorta. Thorax 1968;23:338-9.

2. David TE, Coselli JS, Khoury GE, et al. Aortic valve repair. Semin Thorac Cardiovasc Surg 2015;27:271-87.

3. Gott VL, Greene PS, Alejo DE, et al. Replacement of the aortic root in patients with Marfan's syndrome. N Engl J Med 1999;340:1307-13.

4. Yacoub MH, Gehle P, Chandrasekaran V, et al. Late results of a valve-preserving operation in patients with aneurysms of the ascending aorta and root. J Thorac Cardiovasc Surg 1998;115:1080-90.

5. David TE, Feindel CM. An aortic valve-sparing operation for patients with aortic incompetence and aneurysm of the ascending aorta. J Thorac Cardiovasc Surg 1992;103:61721; discussion 622 .

6. Coselli JS, Volguina IV, LeMaire SA, et al. Early and 1-year outcomes of aortic root surgery in patients with Marfan syndrome: a prospective, multicenter, comparative study. J Thorac Cardiovasc Surg 2014;147:1758-66, 1767.e1-4.

7. Volguina IV, Miller DC, LeMaire SA, et al. Valve-sparing and valve-replacing techniques for aortic root replacement in patients with Marfan syndrome: Analysis of early outcome. J Thorac Cardiovasc Surg 2009;137:1124-32.
Cite this article as: Coselli JS, Weldon SA, Preventza O, de la Cruz KI, LeMaire SA. Valve-sparing versus composite root replacement procedures in patients with Marfan syndrome. Ann Cardiothorac Surg 2017;6(6):692-696. doi: 10.21037/ acs.2017.11.02 